\title{
Angular Momentum Transport in Convectively Unstable Shear Flows
}

\section{Citation}

Käpylä, Petri J., Axel Brandenburg, Maarit J. Korpi, Jan E. Snellman, and Ramesh Narayan. 2010. "Angular Momentum Transport in Convectively Unstable Shear Flows." The Astrophysical Journal 719 (1) (July 16): 67-76. doi:10.1088/0004-637x/719/1/67.

\section{Published Version}

doi:10.1088/0004-637X/719/1/67

\section{Permanent link}

http://nrs.harvard.edu/urn-3:HUL.InstRepos:26526405

\section{Terms of Use}

This article was downloaded from Harvard University's DASH repository, and is made available under the terms and conditions applicable to Other Posted Material, as set forth at http:// nrs.harvard.edu/urn-3:HUL.InstRepos:dash.current.terms-of-use\#LAA

\section{Share Your Story}

The Harvard community has made this article openly available.

Please share how this access benefits you. Submit a story.

\section{Accessibility}




\title{
ANGULAR MOMENTUM TRANSPORT IN CONVECTIVELY UNSTABLE SHEAR FLOWS
}

\author{
Petri J. Käpylä ${ }^{1,2}$, Axel Brandenburg ${ }^{2,3}$, MaArit J. Korpi ${ }^{1}$, Jan E. Snellman ${ }^{1}$, And Ramesh Narayan ${ }^{4}$ \\ 1 Department of Physics, Division of Geophysics and Astronomy, FI-00014 University of Helsinki, Finland \\ 2 NORDITA, AlbaNova University Center, Roslagstullsbacken 23, SE-10691 Stockholm, Sweden \\ 3 Department of Astronomy, Stockholm University, SE-10691 Stockholm, Sweden \\ 4 Harvard-Smithsonian Center for Astrophysics, 60 Garden Street, MS-51, Cambridge, MA 02138, USA \\ Received 2010 March 4; accepted 2010 June 7; published 2010 July 16
}

\begin{abstract}
Angular momentum transport due to hydrodynamic turbulent convection is studied using local three-dimensional numerical simulations employing the shearing box approximation. We determine the turbulent viscosity from nonrotating runs over a range of values of the shear parameter and use a simple analytical model in order to extract the non-diffusive contribution ( $\Lambda$-effect) to the stress in runs where rotation is included. Our results suggest that the turbulent viscosity is on the order of the mixing length estimate and weakly affected by rotation. The $\Lambda$-effect is non-zero and a factor of 2-4 smaller than the turbulent viscosity in the slow rotation regime. We demonstrate that for Keplerian shear, the angular momentum transport can change sign and be outward when the rotation period is greater than the turnover time, i.e., when the Coriolis number is below unity. This result seems to be relatively independent of the value of the Rayleigh number.
\end{abstract}

Key words: accretion, accretion disks - convection - stars: rotation - Sun: rotation - turbulence

Online-only material: color figures

\section{INTRODUCTION}

Turbulence due to the convective instability is thought to account for much of the angular momentum transport in the outer layers of the Sun and other stars with convection zones (e.g., Rüdiger 1989; Rüdiger \& Hollerbach 2004, p. 47). In the presence of turbulence, the fluid mixes efficiently and diffusion processes occur much faster than in its absence. This effect is usually parameterized by a turbulent viscosity $v_{\mathrm{t}}$ that is much larger than the molecular viscosity $v$. Often the value of $v_{\mathrm{t}}$ is estimated using simple mixing length arguments with $v_{\mathrm{t}}=u_{\mathrm{rms}} l / 3$, where $u_{\mathrm{rms}}$ is the rms velocity of the turbulence and $l=\alpha_{\mathrm{MLT}} H$ where $\alpha_{\mathrm{MLT}}$ is a parameter of the order unity and $H$ is the vertical pressure scale height. Numerical results from simpler fully periodic isotropically forced systems suggest that the mixing length estimate gives the correct order of magnitude of turbulent viscosity (e.g., Yousef et al. 2003; Käpylä et al. 2009b; Snellman et al. 2009). However, it is important to compute $v_{\mathrm{t}}$ from convection simulations in order to see whether the results of the simpler systems carry over to convection. Furthermore, it is of interest to study whether the small-scale turbulent transport can be understood in the light of simple analytical closure models that can be used in subgrid-scale modeling. Measuring $v_{\mathrm{t}}$ and its relation to averaged quantities, such as correlations of turbulent velocities, is one of the main purposes of our study.

In addition to enhanced viscosity, turbulence can also lead to non-diffusive transport. The $\alpha$-effect (e.g., Krause \& Rädler 1980), responsible for the generation of large-scale magnetic fields by helical turbulence, is one of the most well-known non-diffusive effects of turbulence. An analogous effect exists in the hydrodynamical regime and is known as the $\Lambda$-effect (Krause \& Rüdiger 1974). The $\Lambda$-effect is proportional to the local angular velocity and occurs if the turbulence is anisotropic in the plane perpendicular to the rotation vector (Rüdiger 1989). The existence of the $\Lambda$-effect has been established numerically from convection simulations (e.g., Pulkkinen et al. 1993; Chan
2001; Käpylä et al. 2004; Rüdiger et al. 2005) and simpler homogeneous systems (Käpylä \& Brandenburg 2008).

If, however, both shear and rotation are present, it is difficult to disentangle the diffusive and non-diffusive contributions. This is particularly important in the case of accretion disks where the sign of the stress determines whether angular momentum is transported inward or outward. Convection is commonly not considered as a viable angular momentum transport mechanism in accretion disks since several studies have indicated that the transport owing to convection occurs inward (e.g., Cabot \& Pollack 1992; Ryu \& Goodman 1992; Stone \& Balbus 1996; Cabot 1996; Rüdiger et al. 2002). Furthermore, in an influential paper, Stone \& Balbus (1996, hereafter SB96) presented numerical simulations of hydrodynamic convection where the transport was indeed found to be small and directed inward on average. This result was used to provide additional evidence for the importance of the magnetorotational instability (Balbus \& Hawley 1991) as the main mechanism providing angular momentum transport in accretion disks. Although we agree with the conclusion that hydrodynamic turbulence is ineffective in providing angular momentum transport, we are concerned about the generality of the result of SB96. There are now some indications that hydrodynamic turbulence may not always transport angular momentum inward (cf. Lesur \& Ogilvie 2010).

In order to approach the problem from a more general perspective, Snellman et al. (2009) studied isotropically forced turbulence under the influence of shear and rotation and found that the total stress, corresponding to the radial angular momentum transport in an accretion disk, can change sign as rotation and shear of the system are varied in such a way that their ratio remains constant. They found that the stress is positive for small Coriolis numbers, corresponding to slow rotation. In what follows, we show that outward transport is also possible for convection in a certain range of Coriolis numbers. When rotation is slow, the Reynolds stress is positive, corresponding to outward transport. In the regime of large Coriolis numbers, the Reynolds 
stress changes sign and is directed inward, as in the study of SB96.

The remainder of the paper is organized as follows. Our numerical model is described in Section 2 and the relevant meanfield description in Section 3. The results and the conclusions are given in Sections 4 and 5, respectively.

\section{MODEL}

Our model is the same as that used in Käpylä et al. (2008), but without magnetic fields. We solve the following set of equations for compressible hydrodynamics,

$$
\begin{gathered}
\frac{\mathcal{D} \ln \rho}{\mathcal{D} t}=-\nabla \cdot \boldsymbol{U} \\
\frac{\mathcal{D} \boldsymbol{U}}{\mathcal{D} t}=-S U_{x} \hat{\boldsymbol{y}}-\frac{1}{\rho} \nabla p+\boldsymbol{g}-2 \boldsymbol{\Omega} \times \boldsymbol{U}+\frac{1}{\rho} \nabla \cdot 2 \nu \rho \mathbf{S} \\
\frac{\mathcal{D} e}{\mathcal{D} t}=-\frac{p}{\rho} \nabla \cdot \boldsymbol{U}+\frac{1}{\rho} \nabla \cdot K \nabla T+2 \nu \mathbf{S}^{2}-Q
\end{gathered}
$$

where $\mathcal{D} / \mathcal{D} t=\partial / \partial t+\left(\boldsymbol{U}+\overline{\boldsymbol{U}}_{0}\right) \cdot \boldsymbol{\nabla}$ is the total advective derivative, $\overline{\boldsymbol{U}}_{0}=(0, S x, 0)$ is the imposed large-scale shear flow, $v$ is the kinematic viscosity, $K$ is the heat conductivity, $\rho$ is the density, $\boldsymbol{U}$ is the velocity, $\boldsymbol{g}$ is the gravitational acceleration, and $\Omega=\Omega_{0} \hat{z}$ is the rotation vector. The fluid obeys an ideal gas law $p=\rho e(\gamma-1)$, where $p$ and $e$ are pressure and internal energy, respectively, and $\gamma=c_{\mathrm{P}} / c_{\mathrm{V}}=5 / 3$ is the ratio of specific heats at constant pressure and volume, respectively. The specific internal energy per unit mass is related to the temperature via $e=c_{\mathrm{V}} T$. The traceless rate-of-strain tensor $\mathbf{S}$ is given by

$$
\mathrm{S}_{i j}=\frac{1}{2}\left(U_{i, j}+U_{j, i}\right)-\frac{1}{3} \delta_{i j} \nabla \cdot \boldsymbol{U},
$$

where commas denote partial differentiation.

We use a three layer, piecewise polytropic stratification with constant gravity $\boldsymbol{g}=-g \hat{z}$. The vertical variation of $g$ in real accretion disks is neglected in our local model. The positions of the bottom of the box, bottom and top of the convectively unstable layer, and the top of the box, respectively, are given by $\left(z_{1}, z_{2}, z_{3}, z_{4}\right)=(-0.85,0,1,1.15) d$, where $d$ is the depth of the convectively unstable layer. Initially the stratification is piecewise polytropic with polytropic indices $\left(m_{1}, m_{2}, m_{3}\right)=(3,1,1)$. The horizontal extent of the box is twice the vertical extent, i.e., $L_{x} / d=L_{y} / d=2 L_{z} / d=4$. The thermal conductivity has a vertical profile that maintains a convectively unstable layer above a stable layer at the bottom of the domain. A prescribed temperature gradient at the base maintains a constant heat flux into the domain. The last term in Equation (3) describes an externally applied cooling with

$$
Q=\frac{e-e_{0}}{\tau_{\mathrm{cool}}(z)}
$$

where $e_{0}$ is the internal energy at $z_{4}$, and $\tau_{\text {cool }}(z)$ is a cooling time which is constant for $z>z_{3}$ and smoothly connects to the lower layer where $\tau_{\text {cool }} \rightarrow \infty$.

We use impenetrable stress-free boundary conditions at the top and bottom boundaries for the velocity

$$
\frac{\partial U_{x}}{\partial z}=\frac{\partial U_{y}}{\partial z}=U_{z}=0 .
$$

The temperature gradient at the bottom of the domain is given by

$$
\frac{\partial T}{\partial z}=\frac{-g / c_{\mathrm{V}}}{\left(m_{1}+1\right)(\gamma-1)},
$$

where $m_{1}=3$ is the polytropic index at $z_{1}$. All quantities are periodic in the $y$-direction, whereas shearing periodic conditions (Wisdom \& Tremaine 1988) are used in the $x$-direction. The same setup has been used in earlier work to model convection in local patches in a star, but here we also apply it to a layer near the surface of an accretion disk. The source of heating in the midplane of the disk is not specified and is instead assumed given. This is appropriate for addressing the more general question about the direction of angular momentum transport once there is convection in the absence of a magnetic field.

We employ the PENCIL CoDE, ${ }^{5}$ which is a high-order finite difference code for solving the compressible MHD equations. The bulk of our simulations were performed at a moderate resolution of $128^{3}$ grid points. In a few cases, we study the behavior of the solutions at higher resolutions (up to $1024^{3}$; see Figure 1 for a snapshot of a high resolution simulation) showing the vertical velocity on the periphery of the domain.

\subsection{Dimensionless Quantities and Parameters}

Non-dimensional quantities are derived such that

$$
d=g=\rho_{0}=c_{\mathrm{P}}=1,
$$

where $\rho_{0}$ is the initial density at $z_{2}$. The units of length, time, velocity, density, and entropy are

$$
[x]=d,[t]=\sqrt{d / g},[U]=\sqrt{d g},[\rho]=\rho_{0},[s]=c_{\mathrm{P}} .
$$

The system is characterized by several non-dimensional parameters. We define the Prandtl number and the Rayleigh number as

$$
\operatorname{Pr}=\frac{v}{\chi_{0}}, \quad \mathrm{Ra}=\frac{g d^{4}}{v \chi_{0}}\left(-\frac{1}{c_{\mathrm{P}}} \frac{d s}{d z}\right)_{0},
$$

where $\chi_{0}=K /\left(\rho_{\mathrm{m}} c_{\mathrm{P}}\right)$ is a reference value for the thermal diffusivity, and $\rho_{\mathrm{m}}$ is the density at the mid layer $z_{\mathrm{m}}=\frac{1}{2}\left(z_{3}-z_{2}\right)$. The entropy gradient, measured at $z_{\mathrm{m}}$ in the non-convecting hydrostatic state, is given by

$$
\left(-\frac{1}{c_{\mathrm{P}}} \frac{d s}{d z}\right)_{0}=\frac{\nabla-\nabla_{\mathrm{ad}}}{H_{\mathrm{P}}},
$$

where $\nabla-\nabla_{\mathrm{ad}}$ is the superadiabatic temperature gradient with $\nabla_{\mathrm{ad}}=1-1 / \gamma, \nabla=(\partial \ln T / \partial \ln p)_{z_{\mathrm{m}}}$, and $H_{\mathrm{P}}$ is the pressure scale height at $z_{\mathrm{m}}$. The amount of stratification is determined by the parameter $\xi_{0}=(\gamma-1) e_{0} /(g d)$, which is the pressure scale height at the top of the domain normalized by the depth of the unstable layer. We use in all cases $\xi_{0}=\frac{1}{3}$, which results in a total density contrast of about 23 . The Mach number in our simulations is of the order of 0.1 or less.

We define the Coriolis and shear numbers, describing the strengths of rotation and shear, respectively, as

$$
\mathrm{Co}=\frac{2 \Omega_{0}}{u_{\mathrm{rms}} k_{\mathrm{f}}}, \quad \mathrm{Sh}=\frac{S}{u_{\mathrm{rms}} k_{\mathrm{f}}},
$$

\footnotetext{
5 http://pencil-code.googlecode.com/
} 


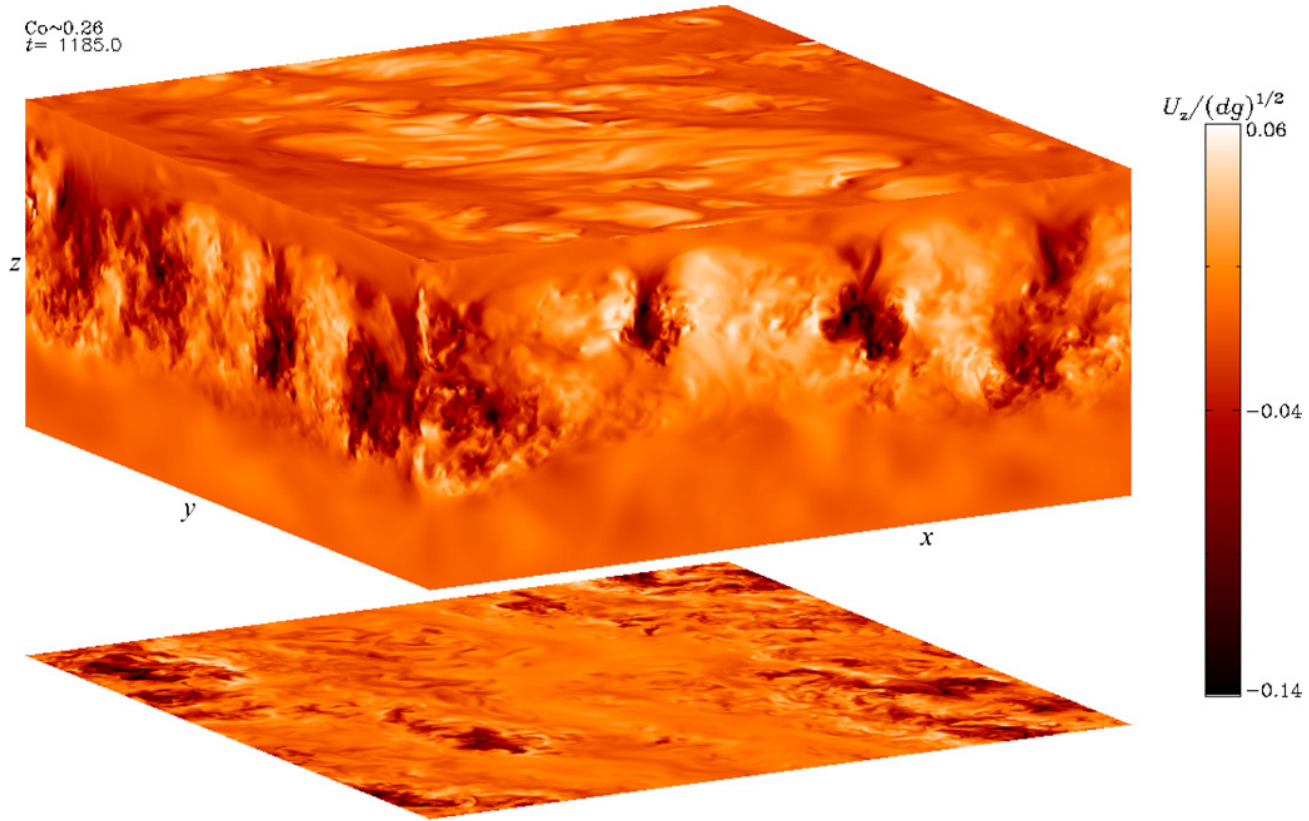

Figure 1. Snapshot of the vertical velocity from run D5 with $\operatorname{Re} \approx 648$. The sides of the box show the periphery of the domain whereas the top and bottom slices show $U_{z}$ at vertical heights $z=0.95 d$ and $z=0$, respectively. Here, Co $=0.26$ and $\mathrm{Sh}=-0.20$. See also http://www.helsinki.fi/ kapyla/movies.html for an animation.

(A color version of this figure is available in the online journal.)

where $u_{\mathrm{rms}}$ is the rms value of the turbulent velocity averaged over the full volume, $k_{\mathrm{f}}=2 \pi / d$ is an estimate of the energy carrying wavenumber, and $d$ is the depth of the convectively unstable layer. The Reynolds number is given by

$$
\operatorname{Re}=\frac{u_{\mathrm{rms}}}{v k_{\mathrm{f}}} .
$$

For accretion disk applications it is convenient to define also the relative shear rate,

$$
q=-\frac{S}{\Omega_{0}},
$$

which describes the rotation profile of the disk, where the local angular velocity varies like $\Omega \propto r^{-q}$. For Keplerian disks we have $q=3 / 2$.

The majority of the simulations ran for $10^{3}$ time units $\left(\Delta t=10^{3} \sqrt{d / g}\right.$ ), which corresponds to roughly 250 convective turnover times; $\tau_{\text {turn }}=\left(u_{\mathrm{rms}} k_{\mathrm{f}}\right)^{-1}$. For the analysis the first 50 turnover times of the simulations were usually discarded in order to minimize the effects of initial transients. The highest resolution runs with $512^{3}$ and $1024^{3}$ grid points were started by remeshing from snapshots of lower resolution runs and ran for 60 and 14 turnover times, respectively.

Error bars are estimated by dividing the time series into three equally long parts and computing averages for each part individually. The largest departure from the average over the full time series is taken to represent the error.

\section{MEAN-FIELD INTERPRETATION}

In mean-field hydrodynamics, the velocity field is decomposed into its mean and fluctuating parts,

$$
\boldsymbol{U}=\bar{U}+\boldsymbol{u},
$$

where the overbar denotes averaging and lowercase $\boldsymbol{u}$ the fluctuation. In the present paper, we consider horizontal averaging so that the mean quantities depend only on $z$. We define the Reynolds stress as

$$
R_{i j}=\overline{u_{i} u_{j}} .
$$

Fluctuations of the density are here ignored for simplicity.

The Reynolds stress is often described in terms of the Boussinesq ansatz which relates the stress to the symmetrized gradient matrix of the large-scale velocity

$$
R_{i j}=-\mathcal{N}_{i j k l} \bar{U}_{k, l}+\ldots,
$$

where the dots indicate higher derivatives of $\overline{\boldsymbol{U}}$ that can occur in the expansion. The expression (17) states that the stress is diffusive in character. In the general case, the fourth rank tensor $\mathcal{N}_{i j k l}$ can have a complicated structure (see, e.g., Rüdiger 1989). However, if we assume that the shear is weak, the simplest description of the horizontal stress generated by our linear shear flow is given by

$$
R_{x y}=-v_{\mathrm{t}}\left(\bar{U}_{x, y}+\bar{U}_{y, x}\right)=-2 v_{\mathrm{t}} \mathrm{S}_{x y}=-v_{\mathrm{t}} S,
$$

where $v_{\mathrm{t}}=v_{\mathrm{t}}(z)$ is the $z$-dependent turbulent viscosity, and the component $S_{x y}$ of the rate-of-strain tensor is not to be confused with the shear rate $S$. To our knowledge, SB96 present the only published results of turbulent viscosity in the absence of rotation as determined from convection simulations with largescale shear, and they only provide a volume averaged quantity for one case. If also rotation is present (cf. Cabot 1996; Lesur \& Ogilvie 2010) the Reynolds stress can no longer be related to turbulent viscosity alone (see below). In the present paper, we study the dependence between stress and shear systematically and estimate the turbulent viscosity coefficient $v_{\mathrm{t}}$.

It turns out that in many applications, Equation (17) is insufficient to describe the stress. For example, according to Equation (18), the Reynolds stress component $R_{\theta \phi}$ derived from observations of sunspot proper motions with the observed surface differential rotation would yield $v_{\mathrm{t}}<0$, which is clearly 
Table 1

Summary of the Runs with Variable $q$

\begin{tabular}{lccccccc}
\hline \hline Run & $q$ & Co & Sh & Re & Ma & $\tilde{R}_{x y}$ & Grid \\
\hline A0 & $\ldots$ & 0 & 0.00 & 28 & 0.036 & $0.003 \pm 0.003$ & $128^{3}$ \\
A1 & $\ldots$ & 0 & -0.04 & 30 & 0.037 & $0.064 \pm 0.020$ & $128^{3}$ \\
A2 & $\ldots$ & 0 & -0.08 & 31 & 0.039 & $0.110 \pm 0.016$ & $128^{3}$ \\
A3 & $\ldots$ & 0 & -0.14 & 33 & 0.041 & $0.152 \pm 0.040$ & $128^{3}$ \\
A4 & $\ldots$ & 0 & -0.17 & 37 & 0.047 & $0.172 \pm 0.040$ & $128^{3}$ \\
\hline B1 & -50.00 & -0.01 & -0.19 & 33 & 0.042 & $0.149 \pm 0.022$ & $128^{3}$ \\
B2 & -25.00 & -0.02 & -0.19 & 34 & 0.042 & $0.151 \pm 0.014$ & $128^{3}$ \\
B3 & -10.00 & -0.04 & -0.20 & 32 & 0.040 & $0.176 \pm 0.017$ & $128^{3}$ \\
B4 & -5.00 & -0.09 & -0.22 & 29 & 0.036 & $0.147 \pm 0.012$ & $128^{3}$ \\
B5 & -2.50 & -0.18 & -0.23 & 28 & 0.035 & $0.119 \pm 0.021$ & $128^{3}$ \\
B6 & -1.00 & -0.49 & -0.25 & 26 & 0.032 & $0.069 \pm 0.010$ & $128^{3}$ \\
B7 & -0.50 & -1.02 & -0.25 & 25 & 0.031 & $0.033 \pm 0.001$ & $128^{3}$ \\
B8 & -0.25 & -2.25 & -0.28 & 23 & 0.028 & $0.014 \pm 0.002$ & $128^{3}$ \\
B9 & -0.10 & -7.67 & -0.38 & 17 & 0.021 & $0.015 \pm 0.005$ & $128^{3}$ \\
\hline C1 & 1.99 & 0.20 & -0.20 & 32 & 0.040 & $0.051 \pm 0.013$ & $128^{3}$ \\
C2 & 1.75 & 0.24 & -0.21 & 30 & 0.038 & $0.039 \pm 0.024$ & $128^{3}$ \\
C3 & 1.50 & 0.30 & -0.22 & 28 & 0.036 & $0.053 \pm 0.003$ & $128^{3}$ \\
C4 & 1.25 & 0.38 & -0.24 & 26 & 0.033 & $0.076 \pm 0.014$ & $128^{3}$ \\
C5 & 1.00 & 0.49 & -0.25 & 26 & 0.032 & $0.066 \pm 0.006$ & $128^{3}$ \\
C6 & 0.75 & 0.67 & -0.25 & 25 & 0.032 & $0.047 \pm 0.007$ & $128^{3}$ \\
C7 & 0.50 & 1.02 & -0.25 & 25 & 0.031 & $0.014 \pm 0.006$ & $128^{3}$ \\
C8 & 0.25 & 2.19 & -0.27 & 23 & 0.029 & $-0.015 \pm 0.005$ & $128^{3}$ \\
C9 & 0.10 & 7.46 & -0.37 & 17 & 0.021 & $-0.036 \pm 0.001$ & $128^{3}$ \\
\hline & & & & & & & \\
\hline
\end{tabular}

Notes. Here, $\mathrm{Ma}=u_{\mathrm{rms}} /(g d)^{1 / 2}$ and $\tilde{R}_{x y}=\left\langle R_{x y}\right\rangle / u_{\mathrm{rms}}^{2}$, where $\left\langle R_{x y}\right\rangle$ is the volume average of the stress over the convectively unstable layer. $\operatorname{Pr}=1$ and $\mathrm{Ra}=10^{6}$ in all runs.

unphysical (see the discussions in Tuominen \& Rüdiger 1989; and Pulkkinen et al. 1993). This motivates the inclusion of a non-diffusive contribution proportional to the rotation of the system (e.g., Wasiutynski 1946), such that

$$
R_{i j}=\Lambda_{i j k} \Omega_{k}-\mathcal{N}_{i j k l} \bar{U}_{k, l}+\ldots,
$$

where $\Lambda_{i j k}$ are the components of the $\Lambda$-effect. This effect is expected to occur in anisotropic turbulence under the influence of rotation (e.g., Rüdiger 1989). In convection the density stratification provides the anisotropy. This is confirmed by numerous simulations of rigidly rotating stratified convection (e.g., Pulkkinen et al. 1993; Chan 2001; Käpylä et al. 2004; Rüdiger et al. 2005). Although additional shear flows are generated in these systems when the gravity and rotation vectors are not parallel or antiparallel, no serious attempt has been made to quantify the turbulent viscosity in convection.

When shear and rotation are both present, it is no longer possible to distinguish between the diffusive and non-diffusive contributions without resorting to additional theoretical arguments. Here, we use a simple analytical approach based on the so-called minimal $\tau$-approximation (see, e.g., Blackman \& Field 2002, 2003) to estimate the contributions from $v_{t}$ and the $\Lambda$-effect.

The idea behind the minimal $\tau$-approximation is to use relaxation terms of the form $-\tau^{-1} R_{i j}$ in the evolution equations for the components of the Reynolds stress $R_{i j}$ as a simple description of the nonlinearities. Using the decomposition (Equation (15)) and the Navier-Stokes equations one can derive equations for the Reynolds stress. For the purposes of the present study it suffices to consider a situation with one spatial dimension, $z$. In this case, the evolution equations can be written
Table 2

Summary of the Runs with Keplerian Shear ${ }^{\mathrm{a}}$

\begin{tabular}{lcccccccc}
\hline \hline Run & Pr & Ra & Co & Sh & Re & Ma & $\tilde{R}_{x y}$ & Grid \\
\hline D1 & 1 & $10^{6}$ & 0.30 & -0.22 & 28 & 0.036 & $0.053 \pm 0.003$ & $128^{3}$ \\
D2 & $\frac{1}{2}$ & $2 \times 10^{6}$ & 0.29 & -0.21 & 59 & 0.037 & $0.053 \pm 0.028$ & $256^{3}$ \\
D3 & $\frac{1}{4}$ & $4 \times 10^{6}$ & 0.27 & -0.20 & 125 & 0.039 & $0.033 \pm 0.004$ & $256^{3}$ \\
D4 & $\frac{1}{10}$ & $10^{7}$ & 0.27 & -0.20 & 315 & 0.039 & $0.018 \pm 0.006$ & $512^{3}$ \\
D5 & $\frac{1}{20}$ & $2 \times 10^{7}$ & 0.26 & -0.20 & 648 & 0.041 & $0.031 \pm 0.010$ & $1024^{3}$ \\
\hline E1 & 1 & $10^{6}$ & 0.01 & -0.01 & 27 & 0.034 & $0.022 \pm 0.021$ & $128^{3}$ \\
E2 & 1 & $10^{6}$ & 0.03 & -0.02 & 26 & 0.033 & $0.032 \pm 0.022$ & $128^{3}$ \\
E3 & 1 & $10^{6}$ & 0.06 & -0.05 & 28 & 0.035 & $0.056 \pm 0.013$ & $128^{3}$ \\
E4 & 1 & $10^{6}$ & 0.12 & -0.09 & 27 & 0.034 & $0.084 \pm 0.007$ & $128^{3}$ \\
E5 & 1 & $10^{6}$ & 0.29 & -0.21 & 29 & 0.037 & $0.045 \pm 0.013$ & $128^{3}$ \\
E6 & 1 & $10^{6}$ & 0.63 & -0.47 & 27 & 0.034 & $0.074 \pm 0.011$ & $128^{3}$ \\
E7 & 1 & $10^{6}$ & 1.32 & -0.99 & 25 & 0.032 & $0.041 \pm 0.011$ & $128^{3}$ \\
E8 & 1 & $10^{6}$ & 4.33 & -3.24 & 20 & 0.025 & $-0.040 \pm 0.003$ & $128^{3}$
\end{tabular}

Note. ${ }^{a}$ Here $q=1.5$ in all runs.

as

$$
\begin{aligned}
\partial_{t} R_{i j}= & -\bar{U}_{z} \partial_{z} R_{i j}-R_{i z} \partial_{z} \bar{U}_{j}-R_{j z} \partial_{z} \bar{U}_{i}-S \delta_{y i} R_{x j} \\
& -S \delta_{y j} R_{x i}-2 \epsilon_{i l k} \Omega_{l} R_{k j}-2 \epsilon_{j l k} \Omega_{l} R_{k i}+N_{i j},
\end{aligned}
$$

where $N_{i j}$ represents the nonlinear terms. As described above, using the minimal $\tau$-approximation as closure model, one substitutes

$$
N_{i j}=-\tau^{-1} R_{i j},
$$

where $\tau$ is a relaxation time, which is here treated as a free parameter that we expect to be close to $\tau_{\text {turn. }}$. In the present paper, however, we will not solve the closure model self-consistently but rather compare the leading terms with the numerical results. A more thorough investigation using the full closure model is postponed to a later study.

\section{RESULTS}

In order to study the Reynolds stress generated by shear and rotation, we perform five sets of simulations summarized in Tables 1 and 2. Our base run is one with $\operatorname{Re} \approx 30, \operatorname{Pr}=1$, and $\mathrm{Co}=\mathrm{Sh}=0$ (run A0). In set A we add only shear and in sets $\mathrm{B}$ and $\mathrm{C}$ we keep the shear constant and vary rotation so that $q$ is negative and positive, respectively. In the remaining sets of calculations, we study the Keplerian shear case $(q=1.5)$ : in set $\mathrm{D}$, we vary $\mathrm{Re}$ and thus Ra with fixed shear and rotation whereas in set E, we vary both Co and Sh with fixed Re.

$$
\text { 4.1. Only Shear, } \mathrm{Sh} \neq 0 \text {, Co }=0
$$

When only shear is present, the turbulent viscosity can be computed from Equation (18) as

$$
v_{\mathrm{t}}=-R_{x y}^{(S)} / S,
$$

where the superscript $S$ indicates that the stress is due to the large-scale shear. By virtue of density stratification, the components of the stress tensor, and hence $v_{t}$, are functions of depth, i.e., $R_{i j}=R_{i j}(z)$ and $v_{\mathrm{t}}=v_{\mathrm{t}}(z)$. We normalize our results with the estimate

$$
v_{\mathrm{t} 0}=\frac{1}{3} \tau \overline{\boldsymbol{u}^{2}} .
$$

Assuming that the Strouhal number,

$$
\mathrm{St}=\tau u_{\mathrm{rms}} k_{\mathrm{f}},
$$




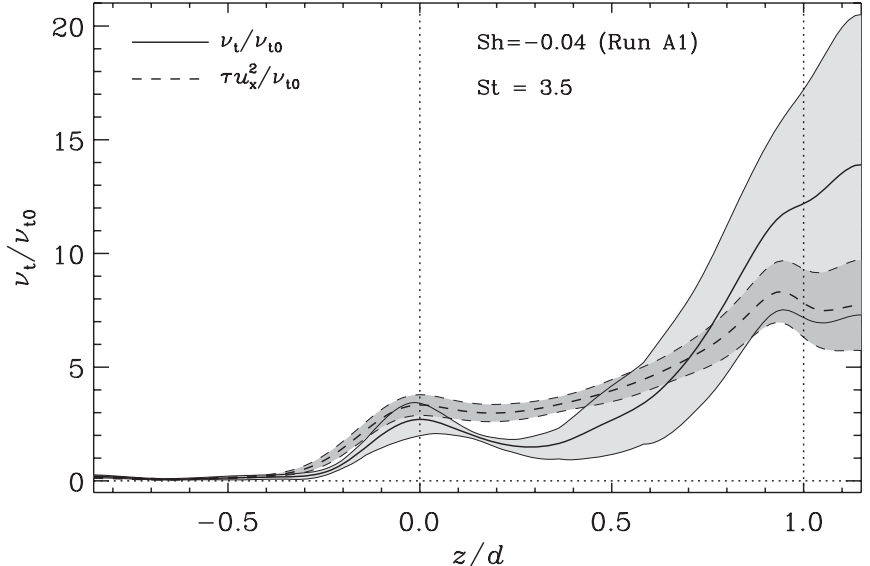

Figure 2. Vertical profile of the turbulent viscosity $v_{\mathrm{t}}$ (thick solid line), computed from Equation (22), and the analytical estimate (thick dashed line) according to Equation (28) for run A1 with $\mathrm{Sh}=-0.04, \mathrm{Co}=0$, and $\mathrm{Re}=30$. The shaded areas show the error estimates. The dotted vertical lines at $z=0$ and $z=d$ denote the bottom and top of the convectively unstable layer, respectively.

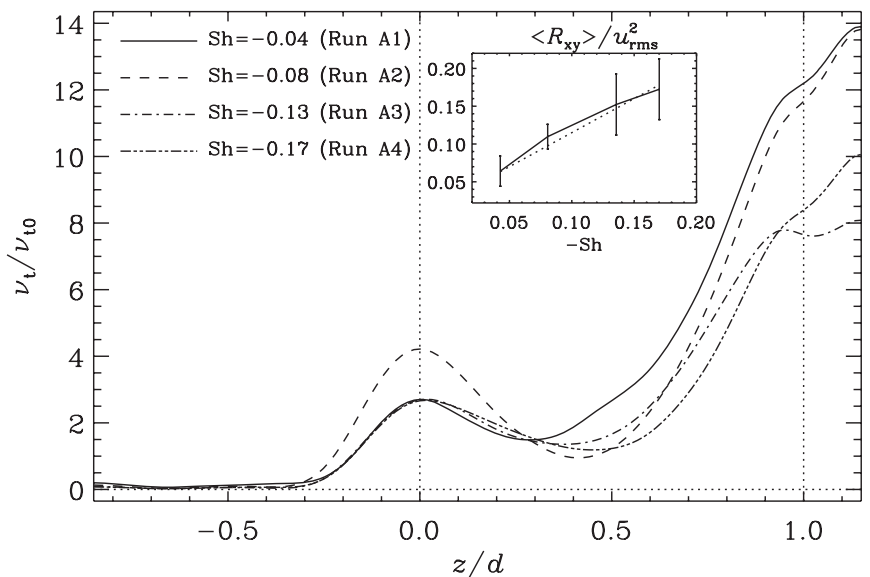

Figure 3. Turbulent viscosity $v_{\mathrm{t}}$ as a function of height for different shear parameters (runs A1-A4). The inset shows $R_{x y}$ averaged over the convectively unstable layer as a function of $\mathrm{Sh}$; dotted line is proportional to $\mathrm{Sh}$

is equal to unity, i.e., $\tau=\tau_{\text {turn }}$, we obtain

$$
v_{\mathrm{t} 0}=\frac{1}{3} u_{\mathrm{rms}} k_{\mathrm{f}}^{-1} \text {. }
$$

Note that if we allow $\mathrm{St} \neq 1$, we have $v_{\mathrm{t} 0}=\frac{1}{3} \mathrm{St} u_{\mathrm{rms}} k_{\mathrm{f}}^{-1}$, and the ratio $v_{\mathrm{t}} / v_{\mathrm{t} 0}$ gives a measure of the Strouhal number. A typical example of $v_{\mathrm{t}}$ is shown in Figure 2. We find that the stress owing to the large-scale shear is always positive, yielding $v_{\mathrm{t}}>0$. The stress $R_{x y}^{(S)}$ increases roughly proportionally to the shear parameter $S$ in the range $0.04<|\mathrm{Sh}|<0.17$ so that the ratio $v_{\mathrm{t}} / v_{\mathrm{t} 0}$ remains approximately constant; see Figure 3. Increasing the shear much beyond our relatively low maximum value leads to large-scale vorticity generation. These flows often grow so strong that the Mach number reaches unity, leading to numerical difficulties. We associate this phenomenon with the "vorticity dynamo" observed in forced turbulence simulations (Yousef et al. 2008; Käpylä et al. 2009b) and several theoretical studies (e.g., Elperin et al. 2003, 2007). Although the large-scale vorticity generation is weak in our runs $\mathrm{A} 1-\mathrm{A} 4$ we see that in the absence of rotation even modest shear increases the rms velocity measurably (see Table 1). However, when rotation is added, this instability vanishes (see, e.g., Snellman et al. 2009).

Our estimate for $v_{\mathrm{t} 0}$ is based on the volume averaged rms velocity and a somewhat arbitrarily defined length scale $1 / k_{\mathrm{f}}$.

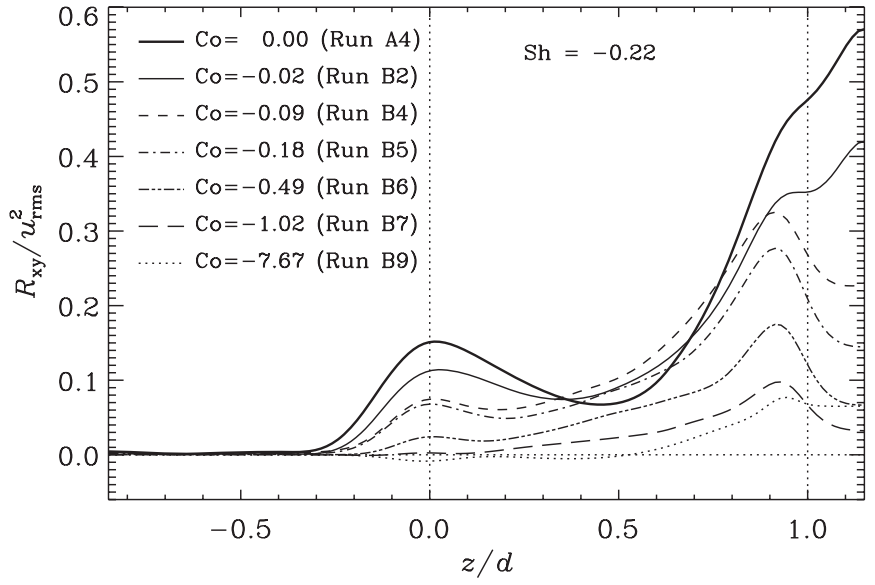

Figure 4. Total stress $R_{x y}$ for different rotation rates using a subset of runs in set $\mathrm{B}$ with negative $q, \mathrm{Sh} \approx-0.22$, and $\mathrm{Re}=30$.

These choices are partly responsible for the large values of $v_{\mathrm{t}} / v_{\mathrm{t} 0}$. In order to obtain a more accurate estimate we derive an evolution equation for $R_{x y}$ using Equation (20)

$$
\partial_{t} R_{x y}=-\bar{U}_{z} \partial_{z} R_{x y}-R_{y z} \partial_{z} \bar{U}_{x}-R_{x z} \partial_{z} \bar{U}_{y}-\overline{u_{x}^{2}} S+N_{x y} .
$$

In general, the first term on the right-hand side is non-zero in the compressible case but, as our Mach numbers are small, $\bar{U}_{z}$ is negligible. Furthermore, the stress components $R_{x z}$ and $R_{y z}$ vanish under the assumption that the imposed shear is the only large-scale velocity component that depends on the horizontal coordinates. Thus, the only terms remaining are

$$
\partial_{t} R_{x y}=-\overline{u_{x}^{2}} S+N_{x y} .
$$

We now ignore the time derivative and apply the minimal $\tau$ approximation, i.e., Equation (21), to obtain

$$
R_{x y}=-\tau \overline{u_{x}^{2}} S=-v_{\mathrm{t}} S .
$$

Note that we use $\tau$ as a fitting parameter when comparing the different sides of the equation. We find that the simple analytical estimate can be fitted with the stress from the simulations when the Strouhal number is in the range 3-4 for all runs in set A; e.g., see the comparison shown in Figure 2 for run A1. Here, for simplicity, we have assumed that $\tau$ has no dependence on $z$ which can contribute to the fact that the curves have somewhat different depth dependencies. Strouhal numbers in the range $1-3$ are in line with previous numerical findings with forced turbulence (e.g., Brandenburg et al. 2004) and convection (e.g., Käpylä et al. 2009a).

\subsection{Shear and Rotation, $\mathrm{Sh}, \mathrm{Co} \neq 0$}

Figures 4 and 5 show the total stress $R_{x y}$ from sets B and C where the imposed shear with $S=-0.05 \sqrt{g / d}$ is kept constant and rotation is varied in a way that $q$ is either negative (set $\mathrm{B}$ ) or positive (set C), respectively. For shear parameters $q>2$, the flow is Rayleigh unstable; thus, we investigate the parameter regime from slightly below 2 down to larger negative values. Note that although the imposed shear is constant, the value of Sh varies somewhat because it is based on the turbulent velocity $u_{\mathrm{rms}}$ which itself is a function of shear and rotation.

We find that for negative $q$ (Figure 4), the stress decreases monotonically as rotation is increased. For slow rotation, i.e., 


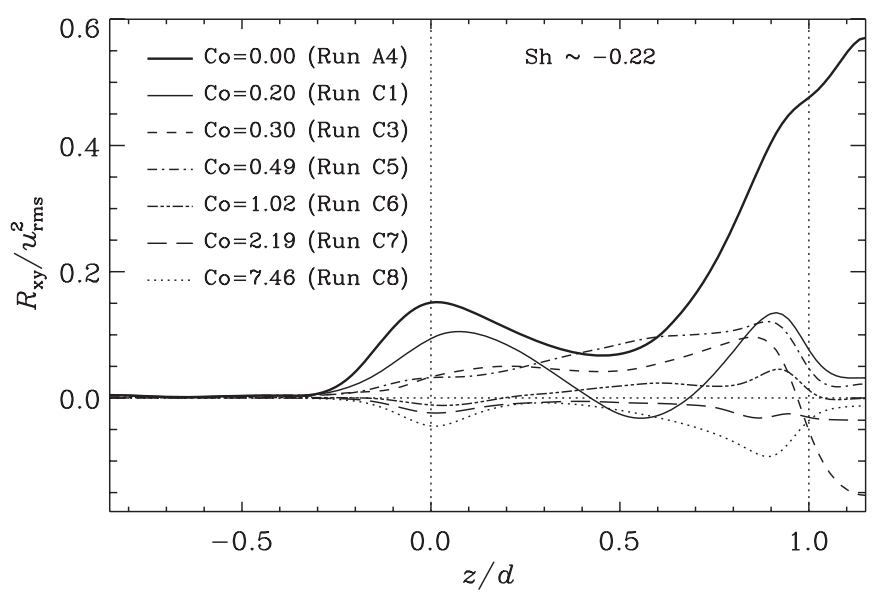

Figure 5. Same as Figure 4, but for a subset of runs from set $\mathrm{C}$ with positive values of $q$ (see the legend).

Co $\gtrsim-0.04$, the differences between the runs are not statistically significant (cf. Figure 2 and Table 1). This is also clear from Figure 6, where we plot the volume average of the stress over the convectively unstable layer as a function of rotation. For more rapid rotation, we interpret the decrease of the stress as the non-diffusive contribution due to the $\Lambda$-effect which is likely a good approximation for slow rotation (see below). The situation is less clear in the case of positive $q$; see Figure 5 and the inset of Figure 6. A contributing factor is that we cannot use arbitrarily small $\Omega$ in the positive $q$ regime because cases with $q>2$ are Rayleigh unstable. However, in the runs that can be done the stress is somewhat decreased in comparison to runs with corresponding $|\mathrm{Co}|$ and negative $q$. For the most rapidly rotating cases in set $\mathrm{C}$ the sign of the stress changes, which is not observed in set $\mathrm{B}$. The sign change suggests that the $\Lambda$-effect dominates over the turbulent viscosity in the rapid rotation regime. Similar asymmetry between the regimes of positive and negative $q$ have also been reported, e.g., by Snellman et al. (2009) and Korpi et al. (2010).

It is interesting to see whether simple analytical models can reproduce the simulation results. For example, in the presence of weak shear and rotation, the minimal $\tau$-approximation applied to a homogeneous system with no convection gives (see Appendix A.2 of Snellman et al. 2009)

$$
\left\langle R_{x y}\right\rangle=a_{1} \mathrm{St} \frac{-(\mathrm{Co}+\mathrm{Sh})\left\langle R_{x x}^{(0)}\right\rangle+\mathrm{Co}\left\langle R_{y y}^{(0)}\right\rangle}{1+4 \mathrm{CoSt}^{2}(\mathrm{Co}+\mathrm{Sh})},
$$

where angular brackets denote volume averaging and the superscript 0 refers to a non-rotating and non-shearing reference state. A similar result was obtained earlier for arbitrary shear and rotation by Narayan et al. (1994) with a conceptually different model where individual eddies were treated as particles that scatter off each other. We note that neither of these models is directly applicable to the present system, although treatment of convection can be introduced into the models (e.g., Kumar et al. 1995). However, our purpose here is not to perform a detailed comparison of the closure models with simulations but rather to attain a broad understanding of the system. In Figure 6, we compare the numerical results with the analytical estimate given in Equation (29), keeping the Strouhal number as a free parameter. Furthermore, we have introduced a scaling parameter $a_{1}(=1.7)$ in order to improve the fit.

We find that parameters $\mathrm{St}=1.0$ and $a_{1}=1.7$ produce a good fit to the numerical results for the runs in set $\mathrm{B}$. We have

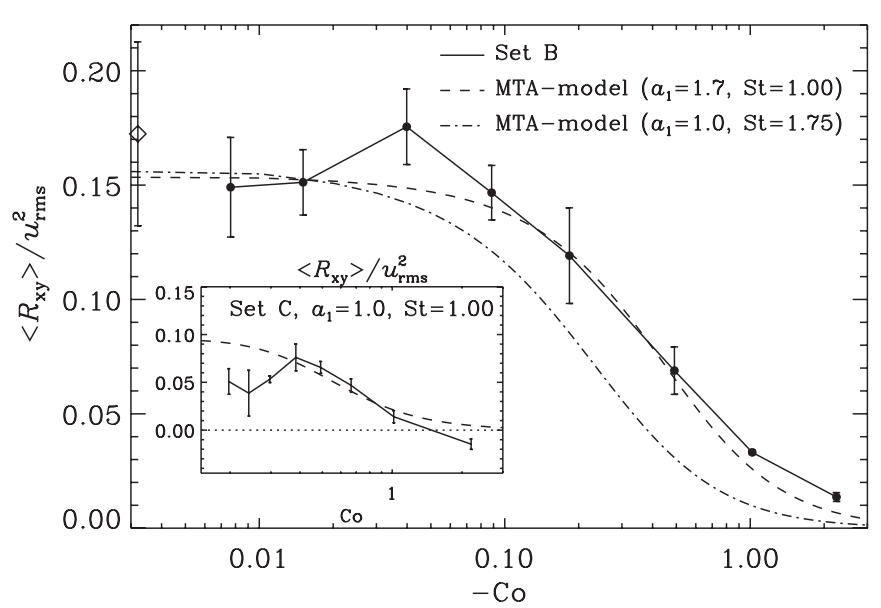

Figure 6. Total stress $R_{x y}$ averaged over the convectively unstable layer for the runs in set B. The dashed and dot-dashed curves show results from the minimal $\tau$-approximation model (Equation (29)). The open symbol on the left denotes the stress for run A4 with no rotation. The inset shows the same representation for the runs in set $\mathrm{C}$.

here normalized our results from Equation (29) by the value of $u_{\text {rms }}$ from the non-rotating run $\mathrm{A} 0$, so a scaling factor $a_{1}$ greater than unity can in principle be understood to reflect the decrease of $u_{\mathrm{rms}}$ as rotation is increased. However, this scaling is not essential since even the unscaled curve shows qualitatively the same behavior. The fit for the runs in set $\mathrm{C}$ is not as successful although the simple model coincides with the simulation data for intermediate values of Co. However, the simulation results fall below the model for $q>1.25$ and the negative stresses for rapid rotation are not captured by the model. The latter is in line with the discovery of Snellman et al. (2009) that the validity of the minimal $\tau$-approximation is limited to the slow rotation regime. The lack of proper parameterization of thermal convection in our simple model is another obvious reason for the differences.

\section{3. $\Lambda$-effect Due to Shear-induced Anisotropy}

In the absence of shear, but including rotation parallel or antiparallel to gravity, turbulence is statistically axisymmetric and there is no asymmetry between the turbulence intensities in the two horizontal directions, i.e., $R_{x x}=R_{y y}$. This implies that there is no horizontal $\Lambda$-effect which, to the lowest order, is proportional to (Rüdiger 1989)

$$
R_{x y}^{(\Omega)} \equiv \Lambda_{\mathrm{H}} \Omega_{0}=2 \Omega_{0} \tau\left(R_{y y}-R_{x x}\right)
$$

Note that the same result is borne out of Equation (20) if we assume that all large-scale flows vanish and allow deviations from axisymmetry, i.e., $R_{x x} \neq R_{y y}$. However, when shear is included, the turbulence becomes anisotropic in the horizontal plane, enabling the generation of a non-diffusive contribution to the stress $R_{x y}$ due to rotation, according to Equation (30). Such contributions have earlier been studied analytically (Leprovost \& Kim 2007, 2008a, 2008b) and numerically (Snellman et al. 2009) for isotropically forced homogeneous turbulence.

When both shear and rotation are present it is not possible to separate the diffusive from the non-diffusive contribution directly. Furthermore, using the diffusive stress from a purely shearing run to extract the non-diffusive one from a run with both the effects is also problematic due to the relatively large errors in the data (cf. Figure 6) which can lead to spurious results. The large errors in the purely shearing runs can possibly 


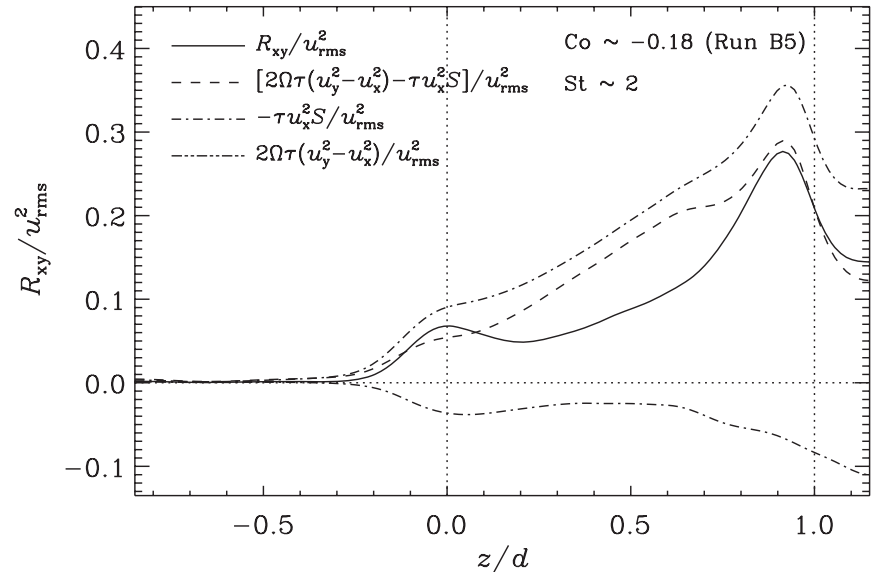

Figure 7. Total stress from the simulation (solid line), total stress from Equation (31) (dashed), diffusive stress from Equation (28) (dot-dashed), and non-diffusive Equation (30) (triple-dot-dashed) for run B5.

be explained by a subcritical vorticity dynamo (cf. Section 4.1). However, if rotation is slow, we can use the simple analytical results of Equations (28) and (30) to express the total stress as

$$
R_{x y}=2 \Omega \tau\left(R_{y y}-R_{x x}\right)-\tau R_{x x} S .
$$

On the other hand, we can express the stress in terms of the $\Lambda$-effect and turbulent viscosity by

$$
R_{x y}=\Lambda_{\mathrm{H}} \Omega-v_{\mathrm{t}} S,
$$

where we now have

$$
\Lambda_{\mathrm{H}}=2 \tau\left(R_{y y}-R_{x x}\right), v_{\mathrm{t}}=\tau R_{x x},
$$

and where we again treat $\tau$ as a fitting parameter. Figure 7 shows an example from run B5. We find that, when $\tau$ corresponding to $\mathrm{St} \approx 2$ is used, the total stress is in broad agreement with Equation (31). In addition to the weaker negative diffusive contribution, corresponding to the turbulent viscosity, we find a non-diffusive part of the opposite sign. Reasonably good fits can be obtained for runs with $|\mathrm{Co}|<1$ with a $\tau$ corresponding to $S t \approx 2$. For more rapidly rotating cases the representation Equation (31) breaks down. Furthermore, in the rapid rotation regime the relevant time scale is the rotation period rather than the turnover time. For the purposes of visualization, and without altering the qualitative character of the results, we consider here volume averages over the convectively unstable layer. Results for runs B1-B7 are shown in Figure 8. We use a fixed $\tau=8 \sqrt{d / g}$, which corresponds to $\mathrm{St} \approx 1.5-2.1$ in these runs. We find that the total stress is fairly well reproduced for runs where $|\mathrm{Co}|$ is below unity. Furthermore, the diffusive contribution stays almost constant as a function of $\mathrm{Co}$, whereas the non-diffusive part is close to zero for $|\mathrm{Co}|<0.1$. The turbulent viscosity, as obtained from Equation (33), shows a weak declining trend as a function of rotation, see the lower panel of Figure 8. The coefficient $\Lambda_{\mathrm{H}}$ has values in the range $(0.5-1) v_{\text {to }}$ for slow rotation. The error estimates increase toward slow rotation, which is consistent with the fact that the nondiffusive stress is small at low Coriolis numbers. These results suggest that the $\Lambda$-effect is non-zero when the anisotropy of the turbulence is induced by the shear flow with a roughly rotationindependent coefficient $\Lambda_{\mathrm{H}}$. However, as our method breaks down when $|\mathrm{Co}| \gtrsim 1$, we cannot study quenching behavior of $v_{\mathrm{t}}$ and the $\Lambda$-effect for rapid rotation.
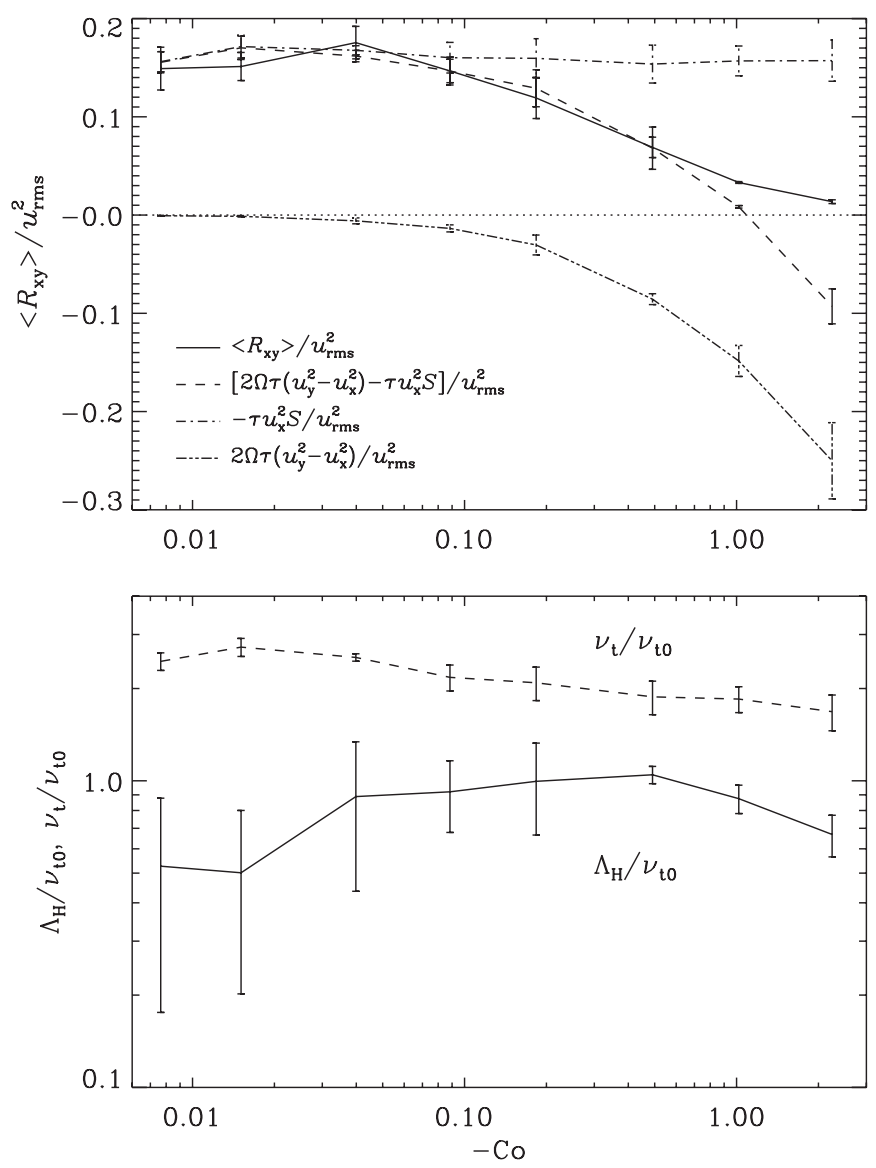

Figure 8. Top panel: total stress for runs B1-B7 (solid line), total stress using Equation (31) (dashed), and the diffusive (dot-dashed) and non-diffusive (tripledot-dashed) contributions according to Equations (28) and (30), respectively. Lower panel: coefficients $\Lambda_{\mathrm{H}}$ and $v_{\mathrm{t}}$ according to Equation (33).

\subsection{Dependence on Reynolds Number}

In set $\mathrm{D}$, we vary the Reynolds number, keeping shear and rotation fixed. Here, we choose $q=1.5$, corresponding to Keplerian shear. Again, the values of Co and Sh are not exactly constant due to the varying $u_{\mathrm{rms}}$; see Table 2 . Figure 9 shows the results for the horizontally averaged stress from the runs in set $\mathrm{D}$. We find that for relatively weak shear and rotation, the stress is positive in the convectively unstable layer-in apparent contradiction with some earlier results (Stone \& Balbus 1996; Cabot 1996) but in accordance with the recent results of Lesur \& Ogilvie (2010). We discuss this issue in the next section in detail.

We also find that the vertical distribution and magnitude of the stress are not much affected when the Reynolds number is increased from 28 to 648, see the inset of Figure 9, where $R_{x y}$ averaged over the convectively unstable layer and time is shown. We note that in addition to the Reynolds number, the Rayleigh number in this set changes by a factor of 20 . However, we have kept the heat conduction, $K$, and thus the input energy flux, constant so that $u_{\mathrm{rms}}$ varies by only $10 \%$ within set D. Had we kept $\operatorname{Pr}=1$, the energy input at the lower boundary would have also decreased by a factor of 20 . This would have resulted in a much lower $u_{\mathrm{rms}}$ and thus proportionally greater values of $\mathrm{Co}$ and Sh. This would have likely produced a very different trend as a function of Ra because Co and Sh are considered as the relevant dimensionless parameters for the Reynolds stress (see, e.g., Snellman et al. 2009). 


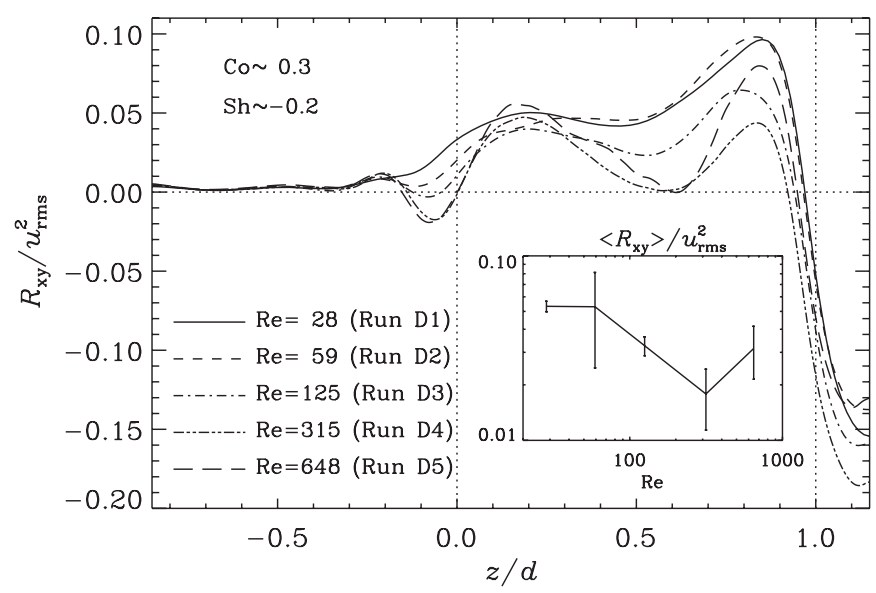

Figure 9. Horizontally averaged stress component $R_{x y}$ from set D with varying Reynolds number and $\mathrm{Co} \approx 0.3$ and $\mathrm{Sh}=-0.2$. The inset shows the stress averaged over the convectively unstable layer as a function of the Reynolds number.

In a recent study, Lesur \& Ogilvie (2010) presented results from Boussinesq convection in an otherwise similar shearing box setup as ours. They report that the stress changes sign from negative to positive in the Keplerian case for strong shear when the Rayleigh number is increased sufficiently. This appears to be in contradiction with our results regarding the dependence on Rayleigh number. However, in their setup the Richardson number (Ri), defined as the negative of the ratio of the squared Brunt-Väisälä frequency and the squared shear rate, is less than unity. This indicates that their models are in the shear-dominated regime whereas in our case only one simulation (run E8) falls in this regime. The low-Ri case at high Rayleigh numbers certainly merits further study.

\subsection{Relation to Accretion Disk Theory}

In their paper, Stone \& Balbus (1996) performed a numerical simulation of convection in a local accretion disk model and found that the total stress is small and on average directed inward. This numerical result based on one simulation and insight drawn from analytical arguments led them to conclude that convection cannot account for the outward angular momentum transport required in astrophysical accretion disks. However, numerical simulations of isotropically forced homogeneous turbulence under the influence of shear and rotation indicate that the total stress can change sign as a function of Co when $q$ is fixed (Snellman et al. 2009). In their study, Snellman et al. (2009) found that, for slow rotation, the stress is positive and changes sign near $\mathrm{Co}=1$. For rapid rotation $(\mathrm{Co} \approx 10)$ the stress appears to drop close to zero. In the context of meanfield hydrodynamics, this can be understood as quenching of the $\Lambda$-effect and turbulent viscosity due to shear and rotation, i.e., $\Lambda_{\mathrm{H}}=\Lambda_{\mathrm{H}}(\Omega, S)$ and $v_{\mathrm{t}}=v_{\mathrm{t}}(\Omega, S)$.

In an effort to study whether these results carry over to convection, we perform a set of runs where we keep $q=1.5$ fixed and vary the values of $\mathrm{Co}$ and Sh. Our results for the horizontally averaged stress are shown in Figure 10. For slow rotation and weak shear, $R_{x y}$ is positive with a maximum value of roughly $10 \%$ of the mean square velocity. As we increase the rotation, the stress decreases and changes sign for $\mathrm{Co} \approx 2$. For Co $=4.33, R_{x y}$ is negative everywhere in the convectively unstable layer. This is when the flow pattern has become markedly anisotropic, as can be seen from visualizations of $U_{\mathrm{z}}$ on (or near) the periphery of the computational domain

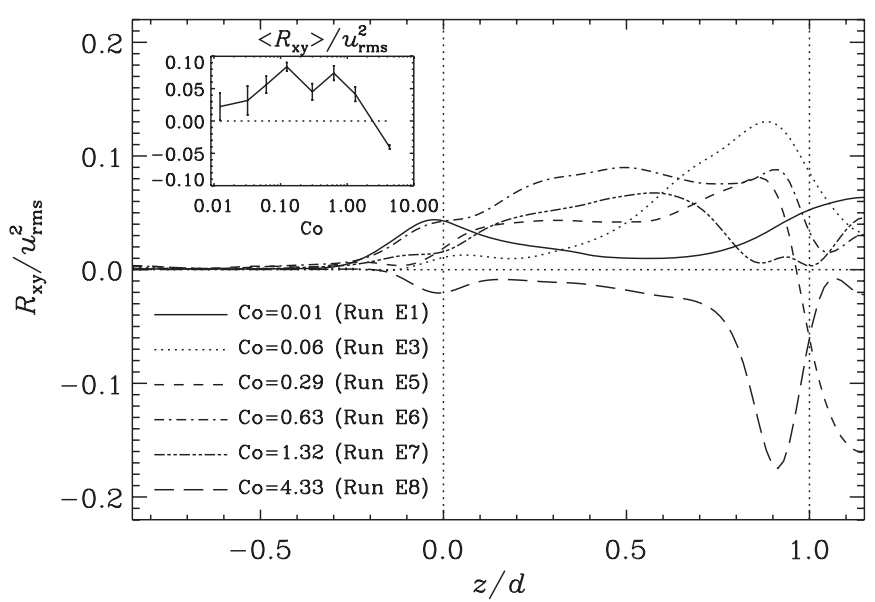

Figure 10. Vertical profiles of $R_{\mathrm{xy}}$ for several values of Co using data for set E. The inset shows $R_{\mathrm{xy}}$ averaged over the convectively unstable layer as a function of Co.

(Figure 11). Indeed, the flow pattern becomes rather narrow in the cross-stream direction, while being roughly unchanged in the streamwise direction. In the rapid rotation regime, the non-axisymmetric structures tend to disappear and promote a two-dimensional flow structure that leads to inward transport (Cabot 1996), which is also visible from our results.

Let us now ask under what conditions can we expect outward angular momentum transport in accretion disks; i.e., when can one expect the Coriolis number to be below unity? In disks, we have $\Omega_{0} H=c_{\mathrm{s}}$, where $H$ is the scale height and $c_{\mathrm{s}}$ is the sound speed. Inserting this into our definition of $\mathrm{Co}$ we obtain from Equation (12)

$$
\mathrm{Co}=\frac{2 \Omega_{0}}{u_{\mathrm{rms}} k_{\mathrm{f}}}=\frac{2 \Omega_{0} H}{u_{\mathrm{rms}}} \frac{1}{k_{\mathrm{f}} H}=\frac{2}{k_{\mathrm{f}} H} \mathrm{Ma}_{\mathrm{t}}^{-1},
$$

where we have defined the turbulent Mach number $\mathrm{Ma}_{\mathrm{t}}=$ $u_{\mathrm{rms}} / c_{\mathrm{s}}$. In our simulations, $k_{\mathrm{f}} H$ is of order unity, which would suggest that we can expect $\mathrm{Co}<1$ only for supersonic turbulence.

A more refined estimate can be obtained by invoking the Shakura-Sunyaev $\alpha$-parameter (Shakura \& Sunyaev 1973), which is introduced as a parameterization of the turbulent viscosity $v_{\mathrm{t}}$ via

$$
v_{\mathrm{t}}=\alpha c_{\mathrm{s}} H .
$$

We can use this parameterization together with Equation (23) to eliminate $u_{\mathrm{rms}}$ in favor of $\alpha$ under the assumption that $v_{\mathrm{t}} \approx v_{\mathrm{t} 0}$. This leads to

$$
\mathrm{Co}=\frac{2 c_{\mathrm{s}}}{3 v_{\mathrm{t}} k_{\mathrm{f}}^{2} H}=\frac{2}{3 \alpha\left(k_{\mathrm{f}} H\right)^{2}} .
$$

In our simulations, we have $H \approx 0.62 d$ at the base of the convectively unstable layer. Together with our estimate $k_{\mathrm{f}}=2 \pi / d$ this yields $k_{\mathrm{f}} H \approx 4$, and therefore Co $\approx(24 \alpha)^{-1}$. We can therefore expect $\mathrm{Co}<1$ for accretion disks where the turbulence is sufficiently vigorous so that $\alpha \gtrsim 0.04$.

We now use our simulations to estimate $\alpha$. In disks, the rate of strain is proportional to $q \Omega_{0}$, so the total turbulent stress is given by

$$
T_{x y}=v_{\mathrm{t}} q \Omega_{0},
$$

where, in the absence of any other stresses such as from magnetic fields, the total stress per unit mass is given by

$$
T_{x y}=R_{x y} \equiv \overline{u_{x} u_{y}} .
$$



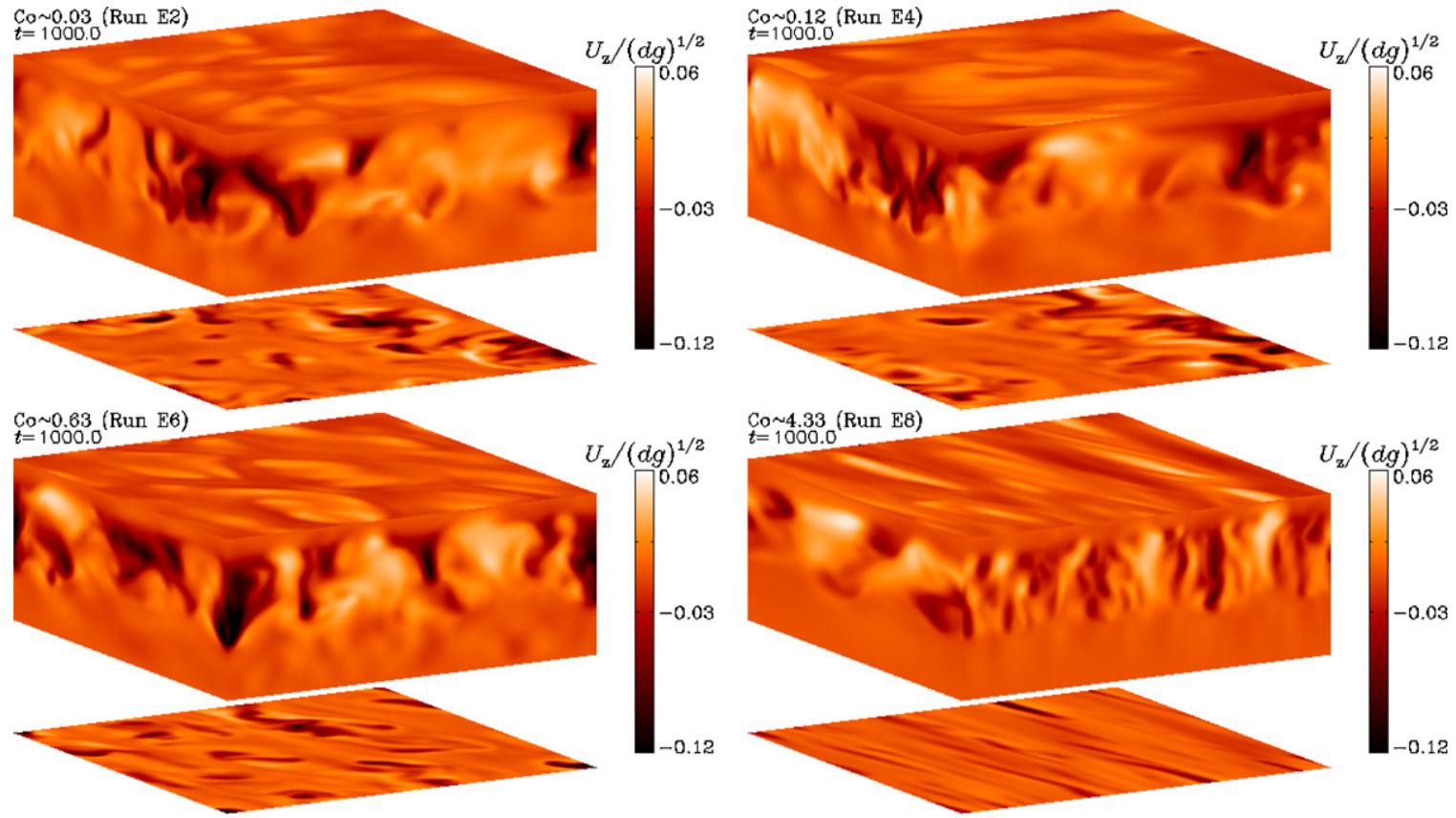

Figure 11. Effect of increasing rotation and shear rates on the visual appearance of the vertical velocity $U_{\mathrm{Z}}$ (runs E2, E4, E6, and E8). All runs are for Keplerian shear, i.e., $q=3 / 2$ and hence $\mathrm{Sh} / \mathrm{Co}=-3 / 4$, the resolution is $128^{3}$ mesh points and the Reynolds number varies between 20 and 30 . (A color version of this figure is available in the online journal.)

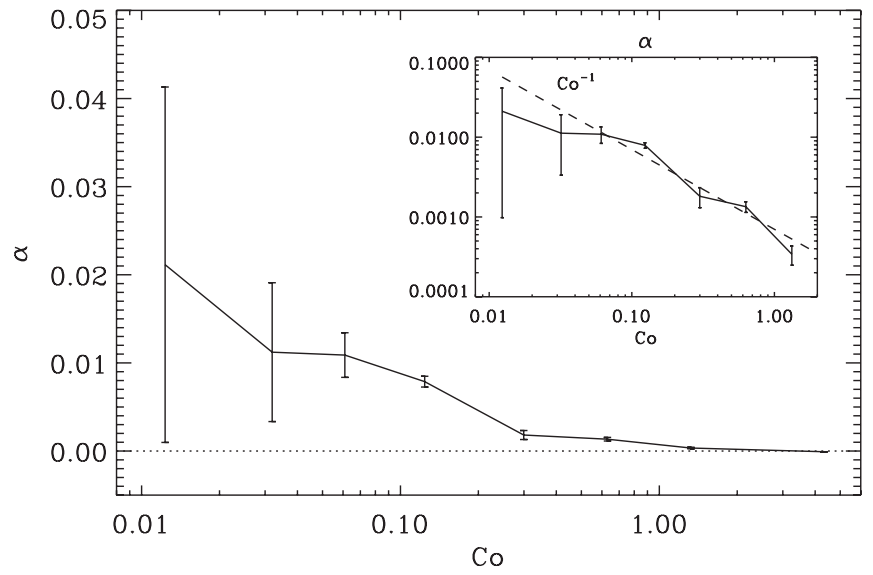

Figure 12. Viscosity parameter $\alpha$ according to Equation (39) for the runs in set E. The inset shows the same in log-log scales. The dashed line shows a scaling inversely proportional to the Coriolis number for reference.

Combining Equations (35) and (37) gives

$$
\alpha=\frac{T_{x y}}{q \Omega_{0} c_{\mathrm{s}} H} .
$$

We compute $T_{x y}$ and hence $\alpha$ as volume averages over the convectively unstable region. For the normalization factor, we take conservative estimates of $c_{\mathrm{s}}$ and $H$ from the bottom of the convectively unstable layer. We find that for slow rotation, i.e., Co $<0.2, \alpha$ is roughly constant with a value of the order of 0.01 (see Figure 12). For more rapid rotation $\alpha$ decreases and eventually changes sign. The points in the range $\mathrm{Co} \approx 0.06-1$ are consistent with a scaling inversely proportional to the Coriolis number which is also suggested by Equation (36). We find that for $\mathrm{Co} \approx 1$ we have $\alpha \approx 4 \cdot 10^{-4}$ which is two orders of magnitude smaller than the estimate derived above.

Another problem facing the suggestion that convection might drive the angular momentum transport in accretion disks is that without an internal heat source in the disk, convection is not self-sustained (cf. SB96). However, many disks are likely to be susceptible to the magnetorotational instability which can extract energy from the shear flow and ultimately deposit it as thermal energy in the disk. If the material in the disk is sufficiently thick optically, the pileup of energy from the magnetorotational instability could render the vertical stratification of the disk convectively unstable.

As alluded to in the introduction, our aim is not to claim that convection is solely responsible for the outward angular momentum transport in accretion disks but to show that, given the right conditions, convection can contribute to outward angular momentum transport.

\section{CONCLUSIONS}

The present results have shown that hydrodynamic convection is able to transport angular momentum both inward and outward, depending essentially on the value of the Coriolis number, in accordance with earlier results from homogeneous isotropically forced turbulence (Snellman et al. 2009). This underlines the importance of considering comprehensive parameter surveys and not relying on demonstrative results from individual case studies. For given value of the Coriolis number, the stress is found to be relatively independent of the value of the Rayleigh number (Section 4.4). By varying shear and rotation rates separately, we have been able to quantify the relative importance of diffusive and non-diffusive contributions to the Reynolds stress tensor. In agreement with earlier work, it turns out that the turbulent kinematic viscosity is of the order of the mixing length estimate and has roughly the same value as the turbulent magnetic diffusivity found earlier for similar runs (Käpylä et al. 2009a). In other words, the turbulent magnetic Prandtl number is around unity, again in accordance with results from simpler systems (e.g., Yousef et al. 2003).

The other important turbulent transport mechanism in rotating turbulent bodies is the $\Lambda$-effect. Although the importance of 
this effect is well recognized in solar and stellar physics (e.g., Rüdiger \& Hollerbach 2004), it is not normally considered in the context of accretion disks. In the present paper, we have been able to quantify its importance for a range of Coriolis numbers by means of a simple analytical model making use of the minimal $\tau$-approximation. For slow rotation the coefficient $\Lambda_{\mathrm{H}}$ is of the order of $v_{\mathrm{t} 0}$ and independent of the Coriolis number. However, once the Coriolis number exceeds a value around unity, our method of separating the turbulent viscosity and the $\Lambda$-effect breaks down, which reinforces the need for a truly independent determination not only of diffusive and nondiffusive contributions to the Reynolds stress, but also of all the components of the full stress tensor.

The authors acknowledge the detailed comments of an anonymous referee. The computations were performed on the facilities hosted by CSC-IT Center for Science Ltd. in Espoo, Finland, which are administered by the Finnish Ministry of Education. We wish to acknowledge the DECI-DEISA network for granting computational resources to the project CONVDYN. Financial support from the Academy of Finland grants no. 121431 (PJK) and 112020 (MJK), the financial support of the Finnish Cultural Foundation (JES), as well as the Swedish Research Council grant 621-2007-4064 and the European Research Council AstroDyn Research Project 227952 (AB) are acknowledged. The authors acknowledge the hospitality of NORDITA during their visits. R.N. acknowledges the financial support from NSF grant AST-0805832.

\section{REFERENCES}

Balbus, S. A., \& Hawley, J. F. 1991, ApJ, 376, 214

Blackman, E. G., \& Field, G. B. 2002, Phys. Rev. Lett., 89, 265007

Blackman, E. G., \& Field, G. B. 2003, Phys. Fluids, 15, L73
Brandenburg, A., Käpylä, P. J., \& Mohammed, A. 2004, Phys. Fluids, 16, 1020 Cabot, W. 1996, ApJ, 465, 874

Cabot, W., \& Pollack, J. B. 1992, Geophys. Astrophys. Fluid Dyn., 64, 97

Chan, K. L. 2001, ApJ, 548, 1102

Elperin, T., Golubev, N., Kleeorin, N., \& Rogachevskii, I. 2007, Phys. Rev.E, 76,066310

Elperin, T., Kleeorin, N., \& Rogachevskii, I. 2003, Phys. Rev. E, 68, 016311

Käpylä, P. J., \& Brandenburg, A. 2008, A\&A, 488, 9

Käpylä, P. J., Korpi, M. J., \& Brandenburg, A. 2008, A\&A, 491, 353

Käpylä, P. J., Korpi, M. J., \& Brandenburg, A. 2009a, A\&A, 500, 633

Käpylä, P. J., Korpi, M. J., \& Tuominen, I. 2004, A\&A, 422, 793

Käpylä, P. J., Mitra, D., \& Brandenburg, A. 2009b, Phys. Rev. E, 79, 016302

Korpi, M. J., Käpylä, P. J., \& Väisälä, M. S. 2010, Astron. Nachr., 331, 34

Krause, F., \& Rüdiger, G. 1974, Astron. Nachr., 295, 93

Krause, F., \& Rädler, K.-H. 1980, Mean-Field Magnetohydrodynamics and Dynamo Theory (Oxford: Pergamon Press)

Kumar, P., Narayan, R., \& Loeb, A. 1995, ApJ, 453, 480

Narayan, R., Loeb, A., \& Kumar, P. 1994, ApJ, 431, 359

Leprovost, N., \& Kim, E. J. 2007, A\&A, 463, 9

Leprovost, N., \& Kim, E. J. 2008a, Phys. Rev. E, 78, 016301

Leprovost, N., \& Kim, E. J. 2008b, Phys. Rev. E, 78, 036319

Lesur, G., \& Ogilvie, G. I. 2010, MNRAS, 404, L64

Pulkkinen, P., Tuominen, I., Brandenburg, A., Nordlund, Å, \& Stein, R. F. 1993, A\&A, 267, 265

Rüdiger, G. 1989, Differential Rotation and Stellar Convection: Sun and SolarType Stars (Berlin: Akademie)

Rüdiger, G., Egorov, P., \& Ziegler, U. 2005, Astron. Nachr., 326, 315

Rüdiger, G., \& Hollerbach, R. 2004, The Magnetic Universe (Weinheim: Wiley$\mathrm{VCH})$

Rüdiger, G., Tschäpe, R., \& Kitchatinov, L. L. 2002, MNRAS, 332, 435

Ryu, D., \& Goodman, J. 1992, ApJ, 388, 438

Shakura, N. I., \& Sunyaev, R. A. 1973, A\&A, 24, 337

Snellman, J. E., Käpylä, P. J., Korpi, M. J., \& Liljeström, A. J. 2009, A\&A, 505, 955

Stone, J. M., \& Balbus, S. A. 1996, ApJ, 464, 364

Tuominen, I., \& Rüdiger, G. 1989, A\&A, 217, 217

Wasiutynski, J. 1946, Astrophys. Nor., 4, 1

Wisdom, J., \& Tremaine, S. 1988, AJ, 95, 925

Yousef, T. A., Brandenburg, A., \& Rüdiger, G. 2003, A\&A, 411, 321

Yousef, T. A., et al. 2008, Phys. Rev. Lett., 100, 184501 